\title{
Ureteric Stent Use - Part of the Solution and Part of the Problem
}

\author{
Victor G. Ilie $\quad$ Vlad I. Ilie \\ aUrology Department; and bDepartment of Surgery, St Vincent Hospital, Sydney, Australia
}

\section{Key Words}

Ureteric stent • Stent encrustation • Stent symptoms

\begin{abstract}
Background: The aim of the study is to comprehensively report on a single tertiary referral center experience with the use of ureteric stents, assess complication burden and determine risk factors to further inform institutional practice. $\mathbf{M a}$ terials and Methods: The retrospectively analyzed cohorts includes 529 patients treated over a 12 months period. Data regarding details of the index pathology, stent characteristics and complications were collected retrospectively. $\boldsymbol{R e}$ sults: Most stents (58.9\%) were used in the context of stone surgery. Stent encrustation occurred in $14.5 \%$ of patients and sepsis in $4.3 \%$. There was a statistically significant difference between the complications of the stents removed after 90 days and the rest of the cohort. Infectious complications were more frequent in diabetic patients and in patients with chronic renal impairment. At our institution, we managed to avoid forgotten stents and catastrophic complications. Conclusion: Stent complications are the complex outcome of interplay between the foreign body and the collecting system environment, influenced by the nature and timing of surgery. Longer stent dwelling times are associated with increased complications rates, especially in patients with significant comorbidities.

Copyright $\odot 2017$ S. Karger AG, Basel
\end{abstract}

\section{Introduction}

The history of the search for methods of draining the obstructed kidneys or bladder parallels the history of urology. The stents are hollow tubes that drain fluids through their lumen and in a laminar fashion around their outer surface. The name is an eponym for three English dentists who developed a substance for dental impression; Goodwin, in 1972, refined the meaning to a mould for internal fixation [1].

The first reported successful use of a ureteric drainage technique belongs to Gustav Simon (1900) during an open cystotomy procedure, followed closely by Joaquin Albarran (1860-1912), credited with the development of the first ureteric stent. Gibbons introduced the first commercially available rubber stent and finally Finney solved the common problem of stent migration by describing the double $\mathrm{J}$ stent and this design is used in the majority of modern stents [2, 3].

The indications for stent placement fall broadly into 3 categories:

1. Stent placement to promote ureteric healing and prevent complications (as, for example, after pyeloplasty or after ureteric repair/replant). The stent allows alignment of the ureteric wall, decreases the inflammation and the urine extravasation and directs epithelial growth. A success rate of $85 \%$ has been reported in the conservative management of ureteral fistulae using stents [4]. As a safety device, the stent allows for the easy identification of the ureter in difficult pelvic surgery procedures.

Victor G. Ilie

St Vincent Hospital

390 Darlinghurst Road, Darllinghurst

Sydney (Australia)

E-Mail ivictorgeorge@gmail.com 
Table 1. Characteristics of the ideal stent

\begin{aligned} & \hline NO. Characteristics \\ & \hline 1 Easily inserted from any access \\ & 2 Resistant to migration \\ & 3 Well tolerated by the patient \\ & 4 Optimal flow characteristics \\ & 5 Biocompatible \\ & 6 Biodurable \\ & 7 Resistant to encrustation \\ & 8 Non-refluxing \\ & 9 Radio-opaque/easily found with ultrasound \\ & 10 Easily exchanged \\ & 11 Affordable \\ & \hline\end{aligned}

2. Stent placement as an adjunct to stone treatment. The stent is a method of drainage in the acute presentation, allowing for stabilization of the patient or a safety device following endourological procedures.

3. Finally, the stent is an optimal device for maintaining long-term patency of the collecting system, most often in the malignant obstruction setting.

In spite of the expanding role for the device, the main characteristics of the ideal stent remain the same (table 1). It has to be said that none of the stents currently in use meet all these demands.

The aim of the exercise was to characterize the use of ureteric stents in a busy tertiary center and to analyze any potential inferences regarding common complications. The ultimate goal was to inform practice regarding the indications and risk profiling in ureteric stenting. We also analyzed the data pertaining to the period to stent removal, with implications for usage of operating time.

\section{Materials and Methods}

This study was part of an internal surgical audit regarding the rigid and flexible ureteroscopy and the use of ureteric stents in a tertiary referral center. After institutional approval was obtained for a low risk project, the data concerning stent usage was collected retrospectively for the period from September 2014 to September 2015.

All the stents removed or inserted in that period were included; the data pertaining to stents used in post-cystectomy diversions or the antegrade stents inserted by the interventional radiology department was not collected. The list of stents was compiled with the help of the surgical coding department and was compared with the data for the ureteroscopy audit, performed concurrently and spanning the same time period.
Table 2. Patient demographics and stent characteristics

\begin{tabular}{lll}
\hline Variables & Value & Median \\
\hline Total patients & & \\
Age, years & 529 & 53 \\
Gender & $20-91$ & \\
$\quad$ Male & & \\
$\quad$ Female & $346(65.4 \%)$ & \\
Diabetes & $183(34.6 \%)$ & \\
$\quad$ Yes & & \\
$\quad$ No & $68(12.8 \%)$ \\
Therapeutic anticoagulation & $461(78.2 \%)$ \\
Stent size, F & $60(11.3 \%)$ \\
$\quad 4.8$ & $387(73.1 \%)$ \\
$\quad 6$ & $115(21.7 \%)$ \\
$\quad 7$ & $27(5.2 \%)$ \\
Stent length, cm & \\
$\quad 24$ & $222(42.0 \%)$ \\
$\quad 26$ & $186(35.1 \%)$ \\
$\quad 22-30$ & $121(22.9 \%)$ \\
Stent dwell time, days & \\
$\quad<30$ & $159(30.1 \%)$ \\
$\quad 30-90$ & $267(50.5 \%)$ \\
$\quad>90$ & $103(19.4 \%)$ \\
Tethered stents (string) & $70(13.1 \%)$ \\
Long-term stents/stent-dependent & $32(6.1 \%)$ \\
patients & \\
\hline
\end{tabular}

All the complications were recorded, including intra-operative complications related to stent insertion or stent removal. Baseline renal function was compared to the renal function close to the end of the stent dwell time. Information regarding results of the urine cultures was collected.

We performed a subgroup analysis depending on the stent dwelling time e.g. less than 30 days, 30 to 90 days and more than 90 days. The intervals were based on published data suggesting minimal morbidity below 90 days but significant complications in stent retained longer than 3 months [5].

All stents in our series were inserted with patient under general anesthesia, in lithotomy position and under image intensifier guidance.

\section{Results}

The cohort included 529 patients identified via clinical records. There was a strong male preponderance (346 vs. 183 or 1.9 to 1 ratio), mainly due to higher percentage of male patients in the category of stents inserted for stone disease.

A sizeable cohort of patients were diabetics (12.8\%) and on therapeutic anticoagulation for cardiac, peripheral vascular or cerebrovascular disease $(11.3 \%)$. The most 
Table 3. Indications for stent placement

\begin{tabular}{lc}
\hline Variables & $\mathrm{n}(\%)$ \\
\hline Obstruction/calculus & $316(59.9 \%)$ \\
Obstruction/malignancy & $32(5.2 \%)$ \\
Perioperative/identification ureter & $11(2 \%)$ \\
Ureteric injury & $17(3.2 \%)$ \\
Ureteric reconstruction & $153(28.9 \%)$
\end{tabular}

Table 4. Stent complications

\begin{tabular}{ll}
\hline Variables & $\mathrm{n}(\%)$ \\
\hline Stent migration & $32(6.1 \%)$ \\
Stent encrustation & $77(14.5 \%)$ \\
Hematuria & $46(8.7 \%)$ \\
Sepsis & $23(4.3 \%)$ \\
Unplanned erectile dysfunction reviews & $40(7.6 \%)$ \\
Early removal stent & $13(2.4 \%)$ \\
Impaired renal function & $29(5.4 \%)$ \\
\hline
\end{tabular}

commonly used stent was the $4.8 \mathrm{~F} 24 \mathrm{~cm}$ double $\mathrm{J}$ stent, most stents having a dwell time of 30-90 days. Of the total number, $13.1 \%$ were tethered and removed in the outpatient clinic via the strings (table 2).

The stents were inserted for a variety of reasons (perhaps underlining the nature of our institution, which is a busy university hospital, with a solid presence of all surgical specialties) (table 3 ). The most common reason for the stent insertion was as an adjunct in the acute and elective treatment of urolithiasis (58.9\%) followed by use in the ureteric reconstruction (28.9\%) and in malignant ureteric obstruction (5.2\%). With a busy colorectal and gynecological oncology department, in $2 \%$ of cases the stents were inserted to help with the intra-operative identification of the ureters.

Overall, $18.8 \%$ of patients had one or more complications related to the stent insertion (table 4). Most commonly, the stents were encrusted (14.5\%). Any degree of solid, adherent accretion observed at stent removal was counted as encrustation. The encrustations occurred predominantly in the stents indwelling for more than 3 months ( $76.3 \%$ of the total encrusted stents) versus $8.5 \%$ in the stents retrieved before 30 days. At removal, 5 pa- tients warranted laser ablation of encrustations to allow safe removal, all of them in the group with a dwell time greater than 90 days.

Of the total patients, $8.7 \%$ experienced hematuria, while in $5.4 \%$ the renal function deteriorated (some in the context of stent migration, which occurred in $6.1 \%$ ).

The rate of unplanned presentation to the emergency department in relation to the stent insertion was $7.6 \%$, mainly due to pain, hematuria, sepsis $(4.3 \%)$ or a combination. Overall, 13 stents $(2.4 \%)$ had to be removed earlier than planned, due to sepsis and pain. There were no instances of forgotten stents in our series.

Urine culture results were available in 387 cases pre stent removal. There were significantly less positive urine cultures rate in normal patients $(3.3 \%)$ than in case with diabetes mellitus or chronic renal failure (33.3 and $39.6 \%$ respectively, $\mathrm{p}<0.001)$. For stents removed within 30 days of insertion the positive urine culture rates was $6.8 \%$, increasing to $28.6 \%$ in stents left longer than 90 days $(\mathrm{p}=0.01)$.

\section{Discussion}

The literature abounds with reports on stent related complications. Stent morbidity is very common and goes significantly beyond discomfort and urinary symptoms (stenting was dubbed "a friendly procedure with unfriendly complications"). The stents are easy to use and reliable (there were only six documented failed attempts at stent insertion during the study period, all in the context of large, obstructed calculi and salvaged by insertion of ipsilateral nephrostomies). There were no issues pertaining to insertion or changes of stents in anticoagulated patients.

It is, unfortunately, difficult to comment on the real incidence of stent pain, dysuria, frequency and urgency on retrospective data. All the patients who represented to the emergency department post stent insertion described pain (among other symptoms). In contemporary studies, up to $85-90 \%$ of patients with indwelling ureteral stents reported irritative voiding symptoms, including frequency, urgency and dysuria, as well as flank pain, suprapubic pain and hematuria; almost half of the patients had to alter their daily activities [6]. A questionnaire for stent symptoms was developed (addressing pain, voiding symptoms, work performance, sexual health, overall general health and additional problems) [7] and it can form the base for future analysis of our cohort. Alpha blockade is associated with decreased stent related morbidity 
and smaller studies have shown benefit of anticholinergic medication. Other experimental approach is to decrease bladder urothelium irritation by intravesical submucosal injection of local anesthetic or by developing stents with a soft distal coil [8].

Clearly the most direct action is to avoid stenting in uncomplicated ureteroscopies. The routine use of alpha blockers not widely adopted and in our institution we used tamsulosin sporadically as discharge medication [9].

Microscopic and mild gross hematuria are usually related to urothelial irritation and self limited. Persistent and significant bleeding after prolonged stenting may be due to ureteric arterial fistula, a rare and devastating complication of stenting. In our cohort, the rate of hematuria was $8.7 \%$ but the retrospective collection might have under-reported the hematuria in otherwise fit patients awaiting stone treatment. In 3 cases of radiation cystitis and ureteric stricture, hematuria prompted early cystoscopy and stent change.

Stent encrustation is a potentially challenging complications associated with prolonged dwell time, history of urolithiasis, metabolic and congenital abnormalities, chemotherapy, bacteriuria and pregnancy. No stent is inert within the urinary tract. Encrustation is a result of successive interplay of the stent surface and collecting system environment, with protein absorption onto biomaterial and bacterial organic molecules deposits on protein substrate forming a conditioning film. At this point, bacteria approach and attach with subsequent growth and biofilm formation. Cations are then attracted to the extracellular matrix with crystal precipitation and further self propagation of crystal formation. Polyurethane (PU) is the most common used stent polymer and common uropathogens adhere to PU leading to shelter time to encrustation. Severe encrustation preferentially occurs at the renal and bladder ends of the stent, with the ureteric segment usually involved last secondary to peristaltic wiping. Coating of stent surface can modify these processes. Hyaluronic acid and covalently bound heparin are inhibitors of nucleation, growth and aggregation of salts and its presence leads to delayed encrustation versus non-coated PU stents [10, 11].

Significant encrustation might warrant a multimodal approach for stent management. In or series, there were no instances of forgotten stents, which, in our opinion is the reason we avoided severe complications such as stent fragmentation (reported as high as $30 \%$ in retained stents) [12], catastrophic renal impairment or uretero-arterial fistulae. All stents that required ureteroscopy and laser lithotripsy for removal were in the group with $>$ 90 days dwell time; it is therefore imperative to radiologically assess a patient with long-term stent prior to removal.

Our deduction as to the cause of the avoidance of retained stents is that our institution is the only tertiary referral center for the area and the urology service covers all other local hospitals. The general practitioners have a unique referral direction and the patients return to the center for their definitive stent management.

To better deal with this major patient safety issue, the use of computerized stent tracking system computer based and barcode acquisition systems significantly increases stent monitoring [13].

Urinary tract infections have been reported to occur in $22-34 \%$ of cases, even with prophylactic antibiotics; a negative urine culture does not rule out infection from stent colonization. In our cohort, 387 patients had urine culture result available; stents removed with a flexible cystoscope had no urine sample analyzed. Sepsis occurred exclusively in patients with stents inserted for obstructive calculi. Also, there was a statistically significant difference depending on dwell time and comorbidities; the diabetics and the patients with dwell time in excess of 90 days had significantly more positive urine cultures, as anticipated by previous published data [14].

Dwell time should be minimized to reduce rate of urinary tract infections, especially in patients with diabetes mellitus, chronic kidney disease and diabetic nephropathy [15]. Further data analysis is needed to characterize the infectious complications; a potential future directions is to send stent tip for culture to better capture the stent/ urine/renal tract interaction.

Among the proposed solutions to this common problem is using pharmacologically active agents incorporated into the stents to prevent infection and encrustation, generating activity at the urothelial level with minimal systemic involvement (such as triclosan loaded stent) [16].

\section{Conclusion}

Stent insertion is a procedure that brings numerous advantages. It does, however, interfere with patient's lifestyle and, in worst case scenario, can cause catastrophic complications.

Our review of the stent practice in a busy tertiary referral center shows that major complications can be avoided if the number of forgotten stents is minimized. 
The situation of our center is quite idiosyncratic in that it is the unique tertiary center for the area. Nevertheless, all efforts need to be made in the direction of medical records, tracking software and patient education arrangements to avoid this serious patient safety issue.

Serious infectious and encrustation related complications can be minimized by decreasing stent dwell time, especially in patients with concurrent diabetes and renal impairment.

\section{Acknowledgements}

Authors would like to acknowledge the senior surgeons with the Urology Department at John Hunter Hospital, Newcastle for kindly providing access to their data: Dr. PJ Ainsworth, Dr. T Doyle, Dr. P Chong, Dr. N McLeod, and Dr. A Tiu.

Authors would also like to acknowledge the significant help with the statistical data analysis from the Department of Medical Statistics with the University of Newcastle, Australia.

\section{References}

1 Lawrentschuk N, Russell JM: Ureteric stenting 25 years on: routine or risky? ANZ J Surg 2004;74:243-247.

2 Gibbons RP, Mason JT, Correa RJ Jr: Experience with indwelling silicone rubber ureteral catheters. J Urol 1974;111:594-599.

3 Finney RP: Experience with new double J ureteral catheter stent. J Urol 1978;120:678681.

4 Chang R, Marshall FF, Mitchell S: Percutaneous management of benign ureteral strictures and fistulas. J Urol 1987;137:1126-1131.

5 Damiano R, Oliva A, Esposito C, De Sio M, Autorino R, D'Armiento M: Early and late complications of double pigtail ureteral stent. Urol Int 2002;69:136-140.

6 Joshi HB, Stainthorpe A, Keeley FX Jr, MacDonagh R, Timoney AG: Indwelling ureteral stents: evaluation of quality of life to aid outcome analysis. J Endourol 2001;15:151-154.
7 Barnes KT, Bing MT, Tracy CR: Do ureteric stent extraction strings affect stent-related quality of life or complications after ureteroscopy for urolithiasis: a prospective, randomised control trial. BJU Int 2014;113:605609.

8 Haleblian G, Kijvikai K, de la Rosette J, Preminger G: Ureteral stenting and urinary stone management: a systematic review. J Urol 2008;179:424-430.

9 Damiano R, Autorino R, De Sio M, Giacobbe A, Palumbo IM, D'Armiento M: Effect of tamsulosin in preventing ureteral stent-related morbidity: a prospective study. J Endourol 2008; 22:651-656.

10 Venkatesan N, Shroff S, Jayachandran K, Doble M: Polymers as ureteral stents. J Endourol 2010;24:191-198.

11 Riedl CR, Witkowski M, Plas E, Pflueger H: Heparin coating reduces encrustation of ureteral stents: a preliminary report. Int J Antimicrob Agents 2002;19:507-510.
12 Dakkak Y, Janane A, Ould-IsmailM T, Ghadouane M, Ameur A, Abbar M: Management of encrusted ureteral stents. Afr J Urol 2012;18:131-134.

13 Lynch MF, Ghani KR, Frost I, Anson KM: Preventing the forgotten ureteral stent: implementation of a web-based stent registry with automatic recall application. Urology 2007; 70:423-426.

14 Kehinde EO, Rotimi VO, Al-Awadi KA, Abdul-Halim H, Al Hunayan A, Pazhoor A: Factors predisposing to urinary tract infection after J ureteral stent insertion. J Urol 2002;167: 1334-1337.

15 Kehinde EO, Rotimi VO, Al-Hunayan A, Abdul-Halim H, Boland F, Al-Awadi KA: Bacteriology of urinary tract infections associated with indwelling $\mathbf{J}$ ureteral stents. $\mathrm{J}$ Endourol 2004;18:891-896.

16 Mendez-Probst CE, Goneau LW, MacDonald KW, Nott L, Seney S, Elwood CN, Lange D, Chew BH, Denstedt JD, Cadieux PA: The use of triclosan eluting stents effectively reduces ureteral stent symptoms: a prospective randomized trial. BJU Int 2012;110:749-754. 